\title{
FUNCTIONS OF EXPONENTIAL TYPE*
}

\author{
BY R. D. CARMICHAEL
}

1. Definition and First Properties. If $f(x)$ is an analytic function which is regular at $x_{0}$ and $x_{1}$, then by expanding $f(x)$ in powers of $x-x_{0}$ and suitably dominating the expansion it is easily shown that

$$
\limsup _{n=\infty}\left|f^{(n)}\left(x_{0}\right)\right|^{1 / n}=\limsup _{n=\infty}\left|f^{(n)}\left(x_{1}\right)\right|^{1 / n},
$$

where the superscripts denote derivatives with respect to $x$. In the proof it is convenient to carry out the argument first for the case in which the first of these superior limits is finite. If these superior limits have the finite value $q,(q \geqq 0)$, then $f(x)$ is an integral function; in such a case we shall say that $f(x)$ is of exponential type $\uparrow$. This terminology is justified by the following fundamental theorem. $\ddagger$

Theorem 1. A necessary and sufficient condition that the integral function $f(x)$ shall be of exponential type $q$ is (1) that numbers $\sigma$ shall exist for which it is true that for every positive number $\epsilon$ there exists a quantity $M$, depending on $\epsilon$ and $\sigma$ in general but independent of $x$, such that for all (finite) values of $x$ we have

$$
|f(x)|<M e^{(\sigma+\epsilon)|x|},
$$

and (2) that $q$ shall be the least possible value for such numbers $\sigma$. Moreover, when $f(x)$ is of exponential type $q$, we have

$$
\left|f^{(n)}(x)\right|<M(q+\epsilon)^{n} e^{(q+\epsilon)|x|}, \quad(n=0,1,2, \cdots),
$$

where $M$ is independent of $x$ and $n$.

The demonstration is readily constructed by aid of (2) which is easily proved by use of the expansion of $f^{(n)}(x)$ in powers of $x$.

* An address delivered by invitation at a meeting of the American Mathematical Society at Cincinnati, December 1, 1933.

$\dagger$ The term exponential type is taken from a paper by G. Polya, Analytische Fortsetzung und konvexe Kurven, Mathematische Annalen, vol. 89 (1923), pp. 179-191.

¥ See p. 361 of a paper by R. D. Carmichael, Summation of functions of a complex variable, Annals of Mathematics, vol. 34 (1933), pp. 349-378. 
The proofs of the following three theorems may also be obtained without difficulty.

Theorem 2. If $f(x)$ and $g(x)$ are two functions of exponential type not exceeding $q$ and if $a$ is a constant, then the function $h(x)$,

$$
h(x)=\int_{a}^{x} g(t) f(x-t) d t
$$

is of exponential type not exceeding $q$.

THEOREM 3. If $f(x)$ is of exponential type $q$ and if the radius $\rho$ of convergence of the power series $\sum a_{k} t^{k}$ is greater than $q$, then the series

$$
\sum_{k=0}^{\infty} a_{k} f^{(k)}(x)
$$

is uniformly convergent in any finite region whatever of the $x$-plane and defines a sum-function $g(x)$ of exponential type not exceeding $q$.

THEOREM 4. Let $f(x)$ be of exponential type not exceeding $q$ and let it have the period $\alpha$. Let $n$ denote the greatest integer not exceeding $|\alpha| q /(2 \pi)$. Then $f(x)$ has the form

$$
f(x)=\sum_{k=-n}^{n} c_{k} e^{2 k \pi i x / \alpha}
$$

where the c's are constants. Moreover every function $f(x)$ of the latter form is of exponential type not exceeding $q$ and is periodic with period $\alpha$.

The following elementary propositions are readily established either by aid of the fundamental Theorem 1 or more directly by means of what is immediately involved in the definition.*

A. If $f(x)$ is of exponential type $q$, then all the derivatives $f^{(n)}(x)$ are of exponential type $q$. A similar proposition holds for all functions obtained from $f(x)$ by repeated indefinite integration with respect to $x$.

* For contributions to the theory of functions of exponential type see (among others) the following: Hilb, Mathematische Annalen, vol. 82 (1920), pp. 1-39, vol. 85 (1922), pp. 89-98; Perron, ibid., vol. 84 (1921), pp. 31-42; Pólya, ibid., vol. 89 (1923), pp. 179-191; Pincherle, Acta Mathematica, vol. 48 (1926), pp. 279-304 (first published in 1888); Hurwitz, ibid., vol. 20 (1897), pp. 285312. 
B. If $f_{1}(x)$ and $f_{2}(x)$ are of exponential type not exceeding $q$, then $a_{1} f_{1}(x)+a_{2} f_{2}(x)$ is of exponential type not exceeding $q$, the quantities $a_{1}$ and $a_{2}$ being constants.

C. If $f_{1}(x)$ and $f_{2}(x)$ are of exponential types $q_{1}$ and $q_{2}$, respectively, and if $q_{1}>q_{2}$, then $f_{1}(x)+f_{2}(x)$ is of exponential type $q_{1}$.

D. If $f(x)$ is of exponential type $q$, then $f(a x+b)$ is of exponential type $|a| q$, when $a$ and $b$ are constants.

E. If $f_{1}(x)$ and $f_{2}(x)$ are of exponential types $q_{1}$ and $q_{2}$, respectively, then the product $f_{1}(x) f_{2}(x)$ is of exponential type not exceeding $q_{1}+q_{2}$.

F. Rational integral functions are of exponential type zero.

G. If $a$ is a constant, the function $e^{a x}$ is of exponential type $|a|$.

2. One-to-One Correspondence with Functions Analytic at a Point. The following theorem appears incidentally in work by Pincherle (loc. cit.) and Hurwitz (loc. cit.).

TheOREm 5. Let $f(x)$ be a function of exponential type $q$ and write

$$
f(x)=a_{0}+a_{1} x+a_{2} x^{2}+\cdots .
$$

Form the function

$$
\phi(t)=\frac{a_{0}}{t}+\frac{a_{1}}{t^{2}}+\cdots+\frac{n ! a_{n}}{t^{n+1}}+\cdots .
$$

The last series converges when $|t|>q$. Let $C$ be a circle about $O$ as center and of radius greater than $q$. Then

$$
f(x)=\frac{1}{2 \pi i} \int_{C} e^{x t} \phi(t) d t .
$$

The proof of the theorem is almost immediate. One has only to replace $\phi(t)$ under the integral sign by its given expansion, multiply this series term by term by $e^{x t}$ and integrate term by term; the result is the power series expansion of $f(x)$.

This theorem exhibits an interesting one-to-one correspondence between functions of exponential type and functions which are analytic at a point and vanish there. Without loss of generality we may take this point of analyticity at infinity. If 
$\phi(t)$ denotes such a function, then it may be written in the form $(4)$; then the associated function $f(x)$ defined by (5) has the expansion (3) and is of exponential type.

When $f(x)$ and $\phi(t)$ are associated in the way indicated it is readily seen that every property of one of the functions is in some way reflected in a corresponding property of the other. It is perhaps most natural to pass from properties of $\phi(t)$ to properties of $f(x)$, using relation (4) as the point of departure for the analysis. One may therefore expect a direct reflection of the properties of $\phi(t)$ in those of $f(x)$. The relation in the opposite order seems to be less direct, but it is certainly fully implied in the indicated correspondence. It appears that very little has been done in developing the indicated correspondence of properties of the two functions. But the problem is certainly a feasible one; in some respects it is no doubt difficult. It is rather intriguing.

3. Expansions in Bernoulli-Hurwitz Functions. A very natural expansion theory of functions of exponential type is afforded by series of Bernoulli-Hurwitz functions (Carmichael, loc. cit., pp. 371-378). The latter functions are extensions of the Bernoulli polynomials $B_{\nu}(x)$ defined by the identity

$$
\frac{t e^{t x}}{e^{t}-1}=\sum_{\nu=0}^{\infty} B_{\nu}(x) \frac{x^{\nu}}{\nu !}
$$

whence we have

$$
B_{\nu}(x)=\frac{\nu !}{2 \pi i} \int_{C} \frac{e^{t x}}{e^{t}-1} \frac{d t}{t^{\nu}}, \quad(\nu=0,1,2, \cdots),
$$

where $C$ is a circle about $O$ as center and of radius less than $2 \pi$. The Bernoulli-Hurwitz functions $B_{v, r}(x)$ we may define (see Hurwitz, loc. cit.) by the relations

$$
B_{\nu, r}(x)=\frac{\nu !}{2 \pi i} \int_{C_{r}} \frac{e^{t x}}{e^{t}-1} \frac{d t}{t^{\nu}}, \quad(\nu, r=0,1,2, \cdots),
$$

where $C_{r}$ denotes the circle of radius $\pi(2 r+1)$ about $O$ as a center. Then we have

$$
B_{\nu, 0}(x)=B_{\nu}(x), \quad(\nu=0,1,2, \cdots),
$$




$$
\begin{array}{r}
B_{\nu, r}(x)=B_{\nu}(x)+\frac{\nu !}{(2 \pi i)^{\nu}} \sum_{k=1}^{r} k^{-\nu}\left\{e^{2 k \pi i x}+(-1)^{\nu} e^{-2 k \pi i x}\right\}, \\
(\nu=0,1,2, \cdots ; r=1,2, \cdots) .
\end{array}
$$

Let us now consider series of the form

$$
\sum_{\nu=0}^{\infty} c_{\nu} B_{\nu, r}(x) / \nu !
$$

where $r$ is a fixed non-negative integer and the coefficients $c_{\nu}$ are independent of $x$. By means of an analysis which is not difficult one is led to the following theorems.

THEOREM 6. If the series (7) converges [converges absolutely] for $x=x_{1}$ and $x=x_{2}$, where $4(r+1) x_{1}$ is not an odd integer and $2(r+1) x_{2}$ and $2(r+1)\left(x_{1}-x_{2}\right)$ are not integers, then in every finite region of the $x$-plane this series converges [converges absolutely] and uniformly. Moreover the series

$$
\sum_{\nu=0}^{\infty} c_{\nu} t^{-\nu}
$$

converges [converges absolutely] for $t= \pm 2 \pi i(r+1)$ and hence also when $|t|>2 \pi(r+1)$.

Theorem 7. No function $F(x)$ can have two distinct expansions in series of the form (7) for the same value of $r$.

THEOREM 8. If a function $F(x)$ has a convergent expansion in the form (7), then $F(x)$ is of exponential type not exceeding $2 \pi(r+1)$.

Theorem 9. Any given function $F(x)$ of exponential type $q$ such that $2 \pi r \leqq q<2 \pi(r+1)$ has an expansion of the form

$$
F(x)=\sum_{k=-r}^{r} a_{k} e^{2 k \pi i x}+\sum_{\nu=1}^{\infty} c_{\nu} B_{\nu, r}(x) / \nu !,
$$

where the a's and c's are (uniquely determined) constants.

CoRollary. The function $F(x)$ has an expansion of the form (8) with $r$ replaced throughout by $r+p$, where $p$ is any nonnegative integer. In each case the finite sum as to $k$ consists of certain terms of the Fourier series for $F(x)$ in the interval $(0,1)$. 
THEOREM 10. Let $F(x)$ be any given function of exponential type $2 \pi(r+1)$. Write

$$
c_{\nu}=F^{(\nu-1)}(1)-F^{(\nu-1)}(0), \quad(\nu=1,2, \cdots) .
$$

Then a necessary and sufficient condition that $F(x)$ shall have an expansion of the form (8) is that each of the following series shall converge:

$$
\sum_{\nu=1}^{\infty} \frac{(-1)^{\alpha \nu} c_{\nu}}{(2 \pi i)^{\nu}(r+1)^{\nu}}, \quad(\alpha=0,1) .
$$

The theorems and corollary in this section afford necessary and sufficient conditions for the expansion of functions in series of Bernoulli-Hurwitz functions. Such series together with a finite number of terms from the indicated Fourier series afford expansions, valid throughout the finite plane, of any given function of exponential type $q$; and no function outside of this class has such expansions. It follows therefore that series of BernoulliHurwitz functions afford very natural means for the representation of functions of exponential type.

From equation (8) and the fact that

$$
B_{\nu, r}(x+1)-B_{\nu, r}(x)=\nu x^{\nu-1}, \quad(\nu=1,2, \cdots),
$$

it follows that

$$
F(x+1)-F(x)=\sum_{\nu=1}^{\infty} c_{\nu} \frac{x^{\nu-1}}{(\nu-1) !}
$$

whence one concludes that the coefficients $c_{\nu}$ in (8) have the values given in (9).

The case $r=0$ of the foregoing expansion theory has been treated by Nielsen* by a method different from that employed by Carmichael in the general case.

4. Other Expansions. A very natural generalization of the expansion theory of the preceding section comes readily to mind. Let $g(t)$ be any function which is analytic at the point $t=0$ and has there the value $g(0)=1$. Then the identity

$$
\frac{e^{t x}}{g(t)}=\sum_{\nu=0}^{\infty} P_{\nu}(x) \frac{t^{\nu}}{\nu !}
$$

* Nielsen, Mathematische Annalen, vol. 59 (1904), pp. 103-109. 
defines the polynomials $P_{\nu}(x)$ of degree $\nu$ for $\nu=0,1,2, \cdots$. If $C$ denotes a sufficiently small circle about $O$ as a center, we have

$$
P_{\nu}(x)=\frac{\nu !}{2 \pi i} \int_{C} \frac{e^{t x}}{g(t)} \frac{d t}{t^{\nu+1}}, \quad(\nu=0,1, \cdots) .
$$

When $g(t)=\left(e^{t}-1\right) / t$, we have $P_{\nu}(x)=B_{\nu}(x)$ and hence are led to the theory of the preceding section. If $g(t)=e^{\alpha t}$, we have $P_{\nu}(x)=(x-\alpha)^{\nu} / \nu$ !, so that the expansion theory in this case is that of power series. These two examples are sufficient to show that there is a great diversity among the expansion theories which may arise from this point of view. The character of the several classes of series will be determined primarily by the additional properties assigned to the function $g(t)$.

In order to have an illustration of the results which may emerge, let us consider the case in which $g(t)$ is further restricted to be analytic in the region $|t|<\rho$ and to be different from 0 in this region except for a zero of order one at a point $\alpha$ of this region. Then if we write

$$
\frac{e^{x t}}{g(t)}-\frac{e^{\alpha x}}{(t-\alpha) g^{\prime}(\alpha)}=\sum_{\nu=0}^{\infty} Q_{\nu}(x) \frac{t^{\nu}}{\nu !},
$$

where $g^{\prime}(t)$ denotes the derivative of $g(t)$ with respect to $t$, we have

$$
Q_{\nu}(x)=P_{\nu}(x)+\nu ! \alpha^{-\nu-1} e^{\alpha x} / g^{\prime}(\alpha) .
$$

By aid of the defining relations for $P_{\nu}(x)$ and $Q_{\nu}(x)$ we readily obtain dominating relations by means of which the following rather remarkable theorem is easily established.

THEOREM 11. In the present case, if the series

$$
\sum_{\nu=0}^{\infty} c_{\nu} P_{\nu}(x)
$$

converges [converges absolutely] at any single point $x=x_{1}$, then in any finite region whatever it converges [converges absolutely] and the convergence is uniform.

The particular case $g(t)=1-t$ yields expansions in terms of the functions 


$$
1+\frac{x}{1 !}+\frac{x^{2}}{2 !}+\cdots+\frac{x^{n}}{n !}
$$

These are sections of the power series expansion of $e^{x}$.

Returning to the general case and differentiating both members of the defining relation for the polynomials $P_{\nu}(x)$, we see that $P_{\nu}(x)=P_{\nu+1}^{\prime}(x)$. These polynomials therefore belong to an interesting class of polynomials.

Let us now consider the case when $g(t)$, with $g(0)=1$, is an integral function having an infinitude of zeros. Let $\lambda_{1}, \lambda_{2}, \cdots$ denote the absolute values of these zeros in ascending order of magnitude, so that $0<\lambda_{1}<\lambda_{2}<\cdots$. Let $C_{r},(r=1,2, \cdots)$, denote a circle about $O$ as center and of radius greater than $\lambda_{r}$ but less than $\lambda_{r+1}$. Let $C_{0}$ denote a circle about $O$ of radius less than $\lambda_{1}$. Define the functions $P_{\nu, r}(x)$ by the relations

$$
P_{\nu, r}(x)=\frac{\nu !}{2 \pi i} \int_{C_{r}} \frac{e^{t x}}{g(t)} \frac{d t}{t^{\nu+1}}, \quad(\nu, r=0,1,2, \cdots) .
$$

These obviously afford generalizations of the Bernoulli-Hurwitz functions. It would be a matter of considerable interest to have developed a comprehensive theory of the expansion of functions in series of the form

$$
\sum_{\nu=0}^{\infty} c_{\nu} P_{\nu, r}(x)
$$

for fixed values of $r$. It would also be of interest to consider the theory for the case when $r$ is a function of $\nu$, as, for instance, the case when $r=\nu$. The first of these investigations will generalize the results of the previous section and will lead to other types of expansions for all functions of exponential type. The latter (see Hurwitz, loc. cit.) will include expansion theories for all integral functions.

Other expansion theories of still more general type would arise from replacing $e^{t x}$ in the foregoing formulas by more general functions of $x$ and $t$. Some classes of cases which thus arise are of considerable interest.

The problems suggested in this section are now being investigated by some of my younger colleagues at the University of Illinois. 
5. Simultaneous Expansions in Composite Power Series. For $n>1$ we consider the question of expanding $n$ integral functions $f_{1}(x), f_{2}(x), \cdots, f_{n}(x)$ simultaneously in composite power series, that is, we consider the problem of representing these functions in the form

$$
f_{\nu}(x)=\sum_{k=0}^{\infty} \sum_{j=1}^{n} c_{j k}\left(x-a_{\nu j}\right)^{k},(\nu=1,2, \cdots, n),
$$

where the coefficients $c_{j k}$ are to be independent of both $x$ and $\nu$. We impose the further condition on the coefficients $c_{j k}$ that they shall be such that the series in the equations

$$
g_{j}(x)=\sum_{k=0}^{\infty} c_{j k} x^{k}, \quad(j=1,2, \cdots, n),
$$

shall converge for all finite values of $x$, thus making the functions $g_{j}(x)$ integral. Furthermore we subject the given constants $a_{\nu j}$ to the condition that the determinant $\Delta(t)$ whose element in $\nu$ th row and $j$ th column is $\exp \left(-a_{\nu j} t\right)$ shall not be identically zero as a function of $t$.

For $n=1$ the problem evidently reduces to the classical problem of expansions in power series. We shall suppose throughout that $n>1$.

We have the following theorems.*

THEOREM 12. If $f_{1}(x), f_{2}(x), \cdots, f_{n}(x)$ are any given integral functions and if the constants $a_{\nu j}$ are such that the determinant $\Delta(t)$ has the property described above, then these functions $f_{\nu}(x)$ have simultaneous expansions of the form (10), where

$$
\lim _{k=\infty}\left|c_{j k}\right| 1 / k=0 \text {. }
$$

THEOREM 13. If the functions $f_{1}(x), f_{2}(x), \cdots, f_{n}(x)$ are of exponential type not exceeding $q$, one at least of them being precisely of type $q$, and if the constants $a_{\nu j}$ are such that the determinant $\Delta(t)$ has the property described above, then the functions $f_{\nu}(x)$ have simultaneous expansions of the form (10) such that the associated functions $g_{j}(x)$ of (11) are of exponential type and indeed such that these functions $g_{j}(x)$ are of exponential type not exceeding $q$, one at least of them being precisely of type $q$.

* R. D. Carmichael, Transactions of this Society, vol. 35 (1933), pp. 1-28. 
It is not difficult to obtain formulas for suitable coefficients $c_{j k}$ to be employed in the expansions (10). Only in exceptional cases is it true that these expansions are unique. Even with the strongest conditions imposed on the coefficients $c_{j k}$ in the latter part of Theorem 13 there is still room for more than one determination of these coefficients. What degree of arbitrariness exists in the determination of these coefficients and what additional conditions might be imposed to render them unique are problems which appear not to have been solved. It seems, however, that they are of such nature as to be amenable to treatment, in large measure at least, by methods which are already existent. In some special cases, particularly for $n=2$, they are not difficult.

As an application of the theorems to the case when $a_{11}=a$, $a_{12}=b, a_{21}=0=a_{22}$, where $a \neq b$, we have the following result. An arbitrary integral function $f(x)$ may be expanded in the form

$$
f(x)=\sum_{k=0}^{\infty}\left\{c_{k}(x-a)^{k}+\gamma_{k}(x-b)^{k}\right\},
$$

where the sums $c_{k}+\gamma_{k},(k=0,1,2, \cdots)$, have any preassigned values subject to the condition that $\left|c_{k}+\gamma_{k}\right|^{1 / k}$ shall approach zero with $1 / k$. This proposition may be given a still more precise form if $f(x)$ is restricted to be a function of exponential type (see Carmichael, loc. cit., p. 19).

The theory just indicated is confined to the case of simultaneous expansions of integral functions. In the case of ordinary power series the natural region of convergence is a circle and the expansion theory exists for all functions which are analytic at a point. This raises the question whether there exists a useful and interesting theory of simultaneous expansions in composite power series in the case of $n$ functions which are analytic in a given finite region. I do not know the answer to this question.

6. Summation of Functions of Exponential Type. By expanding $F(x+\omega)$ and $G(x+\omega)$ in powers of the given constant $\omega$ by means of the Cauchy-Taylor theorem and employing the operator $D$ to denote differentiation with respect to $x$, the equations

$$
F(x+\omega)-F(x)=\omega \phi(x), \quad G(x+\omega)+G(x)=2 \phi(x),
$$


may be written in the symbolic forms

$$
\left(e^{\omega D}-1\right) F(x)=\omega \phi(x), \quad\left(e^{\omega D}+1\right) G(x)=2 \phi(x),
$$

whence one has the symbolic formal solutions

$$
F(x)=\omega\left(e^{\omega D}-1\right)^{-1} \phi(x), \quad G(x)=2\left(e^{\omega D}+1\right)^{-1} \phi(x) .
$$

By aid of the expansions

$$
\begin{gathered}
\frac{\omega}{e^{\omega t}-1}=-\frac{1}{2} \omega+\frac{1}{t}+\sum_{\nu=1}^{m}\left\{\frac{1}{t+2 \nu \pi i / \omega}+\frac{1}{t-2 \nu \pi i / \omega}\right\} \\
\quad+\sum_{\nu=2}^{\infty} B_{\nu}^{(m)} \frac{\omega^{\nu}}{\nu !} t^{\nu-1} \\
\frac{2}{e^{\omega t}+1}=1-\frac{1}{\omega} \sum_{\nu=1}^{m}\left\{\frac{1}{t+(2 \nu-1) \pi i / \omega}+\frac{1}{t-(2 \nu-1) \pi i / \omega}\right\} \\
+\sum_{\nu=1}^{\infty} A_{\nu}^{(m)} \frac{\omega^{\nu}}{\nu !} t^{\nu},
\end{gathered}
$$

where $m$ is a non-negative integer (the finite sums as to $\nu$ being omitted when $m=0$ ), the formal solutions (13) lead to the following formal solutions:

$$
\begin{aligned}
& F(x)=-\frac{1}{2} \omega \phi(x)+\int_{a}^{x} \phi(u) d u \\
&+2 \sum_{\nu=1}^{m} \int_{a}^{x} \cos \frac{2 \nu \pi(u-x)}{\omega} \phi(u) d u+\sum_{\nu=2}^{\infty} B_{\nu}^{(m)} \frac{\omega^{\nu}}{\nu !} \phi^{(\nu-1)}(x), \\
& G(x)=\phi(x)-\frac{4}{\omega} \sum_{\nu=1}^{m} \int_{a}^{x} \cos \frac{(2 \nu-1) \pi(u-x)}{\omega} \phi(u) d u \\
&+\sum_{\nu=1}^{\infty} A_{\nu}^{(m)} \frac{\omega^{\nu}}{\nu !} \phi^{(\nu)}(x),
\end{aligned}
$$

where $a$ is any conveniently chosen constant. We shall call (16) $[(17)]$ the modified Euler-Maclaurin [Boole-Nörlund] sum formula.

We now have the following theorems (Carmichael, Annals of Mathematics, loc. cit., pp. 365, 366).

THEOREM 14. If $\phi(x)$ is of exponential type not exceeding $q$ and if $m$ is the integer defined by the relation 


$$
2 \pi m \leqq|\omega| q<2 \pi(m+1),
$$

then the first equation (12) has a solution $F(x)$ defined by the modified Euler-Maclaurin sum formula (16); and $F(x)$ is of exponential type not exceeding $q$. If $\phi(x)$ is of exponential type $q$, then $F(x)$ is of exponential type $q$.

THEOREM 15. If $\phi(x)$ is of exponential type not exceeding $q$ and $m$ is the integer defined by the relation

$$
(2 m-1) \pi \leqq|\omega| q<(2 m+1) \pi,
$$

then the second equation (12) has a solution $G(x)$ defined by the modified Boole-Nörlund sum formula (17); and $G(x)$ is of exponential type not exceeding $q$. If $\phi(x)$ is of exponential type $q$, then $G(x)$ is of exponential type $q$.

When $\phi(x)$ is of exponential type $q$ we shall say that a solution of the first [second] equation (12) is a principal sum [principal alternating sum ] of $\phi(x)$ if the solution is itself of exponential type $q$.

THEOREM 16. If $\phi(x)$ is of exponential type $q$ and if $m$ is the greatest integer not exceeding $|\omega| q /(2 \pi)$, then the most general principal sum of $\phi(x)$ is the algebraic sum of any particular principal sum (always existent) and the function

$$
\sum_{k=-m}^{m} c_{k} e^{2 k \pi i x / \omega}
$$

where the c's are arbitrary constants.

Theorem 17. If $\phi(x)$ is of exponential type $q$ and if $m$ is the greatest integer not exceeding $\frac{1}{2}+|\omega| q /(2 \pi)$, then the principal alternating sum of $\phi(x)$ is unique when $m=0$ and is otherwise the algebraic sum of a particular principal alternating sum (always existent) and the function

$$
\sum_{k=-m+1}^{m} c_{k} e^{(2 k-1) \pi i x / \omega},
$$

where the c's are arbitrary constants.

The last two theorems for the case $m=0$ are given by Hilb (loc. cit., pp. 90-91). In the general case they are, in view of 
Theorem 1, equivalent to theorems obtained by J. R. Purdy* under hypotheses of a different form.

7. Generalizations of the Preceding Section. We consider the problem of solving the system

$$
\sum_{j=1}^{n} c_{\nu j} g_{j}\left(x+a_{\nu j}\right)=\phi_{\nu}(x), \quad(\nu=1,2, \cdots, n),
$$

of functional equations (generalized difference equations with constant coefficients), where the functions $\phi_{\nu}(x)$ are $n$ given integral functions and the $n$ functions $g_{j}(x)$ are to be determined subject to the requirement that they shall be integral functions. In this system the coefficients $c_{v j}$ and the additive terms $a_{v j}$ in the arguments are given constants.

We define the symbolic operator $E(a)$ by the relation

$$
E(a) \cdot f(x) \equiv f(x+a) .
$$

The product $\alpha E(a) \beta E(b)$ is by definition equal to $\alpha \beta E(a+b)$. We define the symbolic determinant $\Delta$,

$$
\Delta \equiv\left|c_{\nu j} E\left(a_{\nu j}\right)\right|
$$

whose element in $\nu$ th row and $j$ th column is $c_{v j} E\left(a_{v j}\right)$, as the symbolic operator obtained by expanding the determinant formally as if its elements were ordinary algebraic quantities. The expanded determinant may be written as a linear homogeneous function of suitable operators $E$ with constant coefficients. By $h(t)$ we denote the function

$$
h(t)=e^{-x t} \cdot \Delta \cdot e^{x t} .
$$

When $h(t)$ is not identically zero we shall say that system (18) is non-singular. By $A_{v i}$ we shall denote the cofactor of the element in the $\nu$ th row and $j$ th column of $\Delta$. Then $A_{v j}$ is a polynomial in operators $E$ with constants coefficients.

We write the power series expansion of $\phi_{\nu}(x)$ in the form

$$
\phi_{\nu}(x)=\sum_{k=0}^{\infty} s_{\nu k} x^{k} / k !, \quad(\nu=1,2, \cdots, n) .
$$

* J. R. Purdy, The Treatment of Finite Integration by Means of the Cauchy Integral Theorem, (unpublished) Illinois dissertation, 1930. 
When the functions $\phi_{\nu}(x)$ are of exponential type not exceeding $q$, we write

$$
\psi_{\nu}(t)=\frac{s_{\nu 0}}{t}+\frac{s_{\nu 1}}{t^{2}}+\frac{s_{\nu 2}}{t^{3}}+\cdots, \quad(\nu=1,2, \cdots, n) .
$$

These series define the functions $\psi_{\nu}(t)$ when $|t|>q$.

Let $r$ be a positive number exceeding $q$ such that the circle $C_{r}$ of radius $r$ about $O$ as a center passes through no zero of the function $h(t)$. By $A_{v j} e^{x t}$ denote the result of operating with $A_{\nu j}$ on $e^{x t}$ considered as a function of $x$. Form the functions

$$
g_{j}(x)=\frac{1}{2 \pi i} \int_{C_{r}} \sum_{k=1}^{n}\left(A_{k j} e^{x t}\right) \psi_{k}(t) \frac{d t}{h(t)}, \quad(j=1,2, \cdots, n) .
$$

If the functions $\phi_{\nu}(x)$ are of exponential type $q$ or less and at least one of them is of type $q$, then a solution of (18) will be called a principal solution if no function in it is of exponential type exceeding $q$. It is clear that at least one of the functions in the solution must be precisely of type $q$.

Now (see Carmichael, Transactions, loc. cit., p. 7) by aid of certain symbolic processes we are led to the following theorem.

THEOREM 18. When the $\phi_{\nu}(x)$ are functions of exponential type not greater than $q$ and one at least of them is of type $q$, then system (18), when it is non-singular, admits as a principal solution the functions $g_{j}(x)$ defined by (19) for $r=q+\epsilon$, where $\epsilon$ is a small positive quantity such that $h(t)$ has no zero in the ring bounded by $C_{r}$ and the circle $|t|=q$.

By an extension of the argument it may be shown that the non-singular system (18) admits an integral solution whenever the $\phi_{\nu}(x)$ are integral functions.

In the case to which Theorem 18 relates methods are at hand for determining all the principal solutions of (18) and indeed all solutions of exponential type. The singular cases, excluded from Theorem 18, call for additional investigation; but they seem to be amenable to successful treatment. These problems are now being investigated by one of my younger colleagues.

8. Differential Equations of Infinite Order. Let us consider the problem of solving the following linear differential equation of infinite order with constant coefficients: 


$$
a_{0} y+a_{1} y^{\prime}+a_{2} y^{\prime \prime}+\cdots=0, \quad\left(a_{0} \neq 0\right) .
$$

When the coefficients $a_{\nu}$ are given and $y$ is an analytic solution valid at $x=x_{0}$, then the left member of $(20)$ must be a converging series; hence we must have

$$
\limsup _{\nu=0}\left|a_{\nu} y^{(\nu)}\left(x_{0}\right)\right|^{1 / \nu} \leqq 1 .
$$

When this superior limit is 1 there is still doubt concerning the required convergence. Hence, in a first study of (20), it is natural to require that the given superior limit shall be less than 1 . Then it is natural to impose on the given coefficients the condition

$$
\limsup _{\nu=\infty}\left|a_{\nu}\right|^{1 / \nu}=\rho<\infty .
$$

One is thus led to seek solutions $y$ of (20) such that

$$
\limsup _{\nu=\infty}\left|y^{(\nu)}(x)\right|^{1 / \nu}<\frac{1}{\rho}
$$

Such functions are of exponential type. Therefore functions of exponential type are naturally encountered in the study of the simplest differential equations of infinite order.

The central theorem* which emerges in the investigation of the problem thus suggested is the following.

THEOREM 19. In the linear homogeneous differential equation (20) of infinite order, suppose that the given coefficients $a_{\nu}$ are such that the function $F(z)$,

$$
F(z)=a_{0}+a_{1} z+a_{2} z^{2}+\cdots,
$$

is analytic in the region $|z| \leqq q,(q>0)$. Let solutions $y(x)$ be sought of exponential type not exceeding $q$. If $F(z)$ has no root in the region $|z| \leqq q$, the only solution $y(x)$ of the indicated character

* For the theory of differential equations of infinite order the reader may consult the following papers and others referred to in them: Hilb, Mathematische Annalen, vol. 82 (1920), pp. 1-39; vol. 84 (1921), pp. 16-30, and pp. 43-52; vol. 85 (1922), pp. 89-98; Perron, Mathematische Annalen, vol. 84 (1921), pp. 1-15, and pp. 31-42; Ritt, Transactions of this Society, vol. 18 (1917), pp. 27-49; Davis, Annals of Mathematics, vol. 32 (1931), pp. 686-714; Lewis, Transactions of this Society, vol. 35 (1933), pp. 792-823. The results which we present are essentially in the form given by Perron. 
is $y(x) \equiv 0$. If $F(z)$ has $n$ zeros $(n>0)$ in the region $|z| \leqq q$ (multiple zeros multiply counted) and if $P(z), P(z) \equiv z^{n}+g_{1} z^{n-1}+\cdots$ $+g_{n}$, is a polynomial having the same zeros as $F(z)$ in this region (with the same multiplicities), then the solutions $y(x)$ of the indicated character are the same as the solutions of the following equation of finite order:

$$
u^{(n)}+g_{1} u^{(n-1)}+\cdots+g_{n-1} u^{\prime}+g_{n} u=0 .
$$

An elegant and rather comprehensive theory has been developed by Hilb, Perron, and others for the solutions of exponential type admitted by equations of the form

$$
\sum_{\nu=0}^{\infty} g_{\nu}(x) y^{(\nu)}=f(x)
$$

where $f(x)$ is a given function of exponential type and the $g_{\nu}(x)$ are given polynomials of degrees not exceeding a given integer $p$. For the results the reader may conveniently consult Perron (loc. cit.). It is thus seen that some of the most important investigations of the theory of differential equations of infinite order are essentially bound up with the theory of functions of exponential type. In particular, there are classes of cases in which one is able to obtain the general solution of exponential type.

That homogeneous equations even with constant coefficients may have solutions not of exponential type is perhaps to be expected; it is proved by Ritt (loc. cit.) by means of an example. The problem of constructing a comprehensive theory of a class of solutions not of exponential type seems to be difficult.

9. Exponential Sums. An interesting particular class of functions of exponential type is constituted by functions of the form

$$
\sum_{k=0}^{n} A_{k}(x) e^{\alpha x},
$$

where the $\alpha$ 's are constants and the $A$ 's are polynomials in $x$. These functions are rich in properties and have numerous connections of importance. An expository discussion of the distribution of their zeros has been given by R. E. Langer,* with references to the literature. It seems highly probable that many

* Langer, this Bulletin, vol. 37 (1931), pp. 213-239. 
properties of the distribution of these zeros will admit of interesting generalizations to more extended classes of functions of exponential type. The paper of Pólya, already cited, contains results which seem to point the way to a more extended investigation of this problem. If results of this sort could be obtained in a comprehensive form they would have intimate connections with several investigations of marked interest.

10. Expansions in Series of Exponential Functions. The expansions here intended have been investigated by Carmichael (Transactions of this Society, loc. cit., pp. 21-28), on whose work this section is based. Let us denote by $h(t)$ the function

$$
h(t)=c_{1} e^{a_{1} t}+c_{2} e^{a_{2} t}+\cdots+c_{n} e^{a_{n} t}, \quad(n>1),
$$

where the $a$ 's are different constants and the $c$ 's are constants different from zero. Let $P$ be the smallest convex polygon, in the complex plane, containing the points $a_{1}, a_{2}, \cdots, a_{n}$; this polygon may in special cases reduce to a straight line segment. Let $Q$ be the polygon* obtained by reflecting $P$ through the real axis.

Let $C_{1}, C_{2}, \cdots$ be a set of different closed contours in the $x$-plane such that any given point on $C_{j}$ is either interior to $C_{j+1}$ or on $C_{j+1}$ and let these contours have the additional properties prescribed in the memoir cited (Carmichael, loc. cit., p. 23).

Let $\psi(t)$ be any function of $t$ which is analytic at infinity and vanishes there and let us write

$$
\psi(t)=\frac{\gamma_{1}}{t}+\frac{\gamma_{2}}{t^{2}}+\cdots, \quad(|t|>q) .
$$

Let $r$ be an integer such that the contour $C_{r}$ lies entirely within the region of convergence of the series in (22). Form the function $F_{r}(x)$,

$$
F_{r}(x)=\frac{1}{2 \pi i} \int_{C_{r}} e^{x t}\{h(t)\}^{-1} \psi(t) d t .
$$

Then $F_{r}(x)$ is a function of exponential type.

Let $p$ be any positive integer and form the function $F_{r+p}(x)$ by changing $r$ to $r+p$ in (23). Then it may be shown that

$$
\lim _{p=\infty} F_{r+p}(x)=0
$$

\footnotetext{
* Such polygons $P$ and $Q$ have been employed by P6lya (loc. cit.).
} 
when either of the following conditions is satisfied:

(1) when $x$ is in the interior or on the boundary of $P$ and is not a vertex of $P$;

(2) when $x$ is a vertex of $P$ provided in this case that $\gamma_{1}=0$.

Let $S_{r+p}(x)$ denote the negative of the sum of the residues of the function $e^{x t}\{h(t)\}^{-1} \psi(t)$ in the region bounded by the contours $C_{r+p-1}$ and $C_{r+p}$. If the function has no singularity in this region, we shall understand that $S_{r+p}(x)$ is identically zero. In all other cases $S_{r+p}(x)$ is a function of the form $c e^{\alpha x}$ or a sum of a finite number of such functions. Then we have the following theorem (remarkable for the character of the region of convergence of the indicated series).

THEOREM 20. The function $F_{r}(x)$ defined in (23) has the expansion

$$
F_{r}(x)=\sum_{k=1}^{\infty} S_{r+k}(x)
$$

in series of exponential functions, valid for all values of $x$ in the polygon $P$, except that the vertices are to be excluded when $\gamma_{1} \neq 0$. (There are cases when the series has no other points of convergence.)

When $h(t)=e^{t}-1$, the series in (24) is a Fourier series. The polygon $P$ in this case reduces to the interval $(0,1)$ of the real axis.

The foregoing theorem serves to expand in series (24) any function whatever that may be put in the form (23). If $h(0) \neq 0$, it is evident that any given polynomial in $x$ may be put in the form $F_{1}(x)$ by taking $C_{1}$ to be a small circle about $O$ as a center and by choosing $\psi(t)$ properly as a polynomial in $1 / t$. The function $F_{1}(x)+$ constant may also in other cases represent any polynomial whatever in $x$. Hence, in particular, all polynomials have expansions in the form (24), valid in polygons $P$ as indicated.

More specific results may be obtained in the case when

$$
\frac{e^{x t}}{h(t)}=\frac{e^{x t}}{\left(e^{\rho_{1} t}-1\right)\left(e^{\rho_{2} t}-1\right) \cdots\left(e^{\rho_{n} t}-1\right)},
$$

where $\rho_{1}, \rho_{2}, \cdots, \rho_{n}$ are $n$ real or complex constants different 
from zero and such that neither the sum nor the difference of two of them is zero. In this case it is convenient to normalize the problem by means of certain elementary transformations. If $\rho_{k}$ has a negative real part we may replace $\rho_{k}$ by $-\rho_{k}$ by multiplying both numerator and denominator in (25) by $-e^{-\rho_{k} t}$ and then replace $x-\rho_{k}$ by $x$. Hence we may (and we do) take the real part of each $\rho_{k}$ to be non-negative. Then the further conditions on the $\rho$ 's are that they are different from each other and from zero. Then the point zero is on the boundary of the polygon $P$ and the greatest real value of a point in $P$ is the sum of the real parts of the $\rho$ 's. We assume that

$$
-\frac{1}{2} \pi \leqq \arg \rho_{1} \leqq \arg \rho_{2} \leqq \cdots \leqq \arg \rho_{n} \leqq \frac{1}{2} \pi .
$$

It is easy to show that the points $x$ of $P$ are the following:

$$
x=\lambda_{1} \rho_{1}+\lambda_{2} \rho_{2}+\cdots+\lambda_{n} \rho_{n}, \quad\left(0 \leqq \lambda_{k} \leqq 1, k=1,2, \cdots, n\right) .
$$

In what follows we shall suppose that no two of the numbers $\rho_{k}$ have a real ratio.

Now we choose (as we may) the contours $C_{1}, C_{2}, \cdots$ so that $C_{k}$ encloses just $k$ zeros of $h(t)$. Hence the terms $S_{r+k}(x)$ in (24) may all be taken in the form $c e^{\alpha x}$ so that we have to do with expansions of the form

$$
F(x)=\alpha_{00}+\sum_{m=1}^{\infty} \sum_{k=1}^{n}\left(\alpha_{k m} e^{2 m \pi i x / \rho_{k}}+\beta_{k m} e^{-2 m \pi i x / \rho_{k}}\right) .
$$

With each of the functions

$$
\begin{aligned}
& 1, \quad e^{2 m \pi x / \rho_{k}}, e^{-2 m \pi i x / \rho_{k}}, \\
&(k=1,2, \cdots, n ; m=1,2,3, \cdots),
\end{aligned}
$$

let us associate its reciprocal and let us call this associated function the adjoint of the given function. If we multiply any function whatever of the set (27) by the adjoint of any other function in the set, we have a product of the form

$$
\prod_{k=1}^{n} e^{2} l_{k} \pi i x / \rho_{k},
$$

where at least one and not more than two of the integers $l_{k}$ are different from zero. There is a side of the polygon $P$ on which 
$x / \rho_{k}$ ranges from 0 to 1 ; on that side we denote $x / \rho_{k}$ by $\lambda_{k}$. Then

$$
\int_{0}^{1} \int_{0}^{1} \cdots \int_{0}^{1}\left(\prod_{k=1}^{n} e^{2 l_{k} \pi i \lambda_{k}}\right) d \lambda_{1} d \lambda_{2} \cdots d \lambda_{n}=0 .
$$

If a like integral is formed with a function of the set (27) and the adjoint of that function then this corresponding integral has the value 1 . Hence we have conditions of biorthogonality generalizing those pertaining to the case of Fourier series, here arising when $n=1$. Consequently we have a formal method of determining the coefficients in series (6) for a much more extensive class of functions than those for which we have established the validity of such expansions. This suggests the generalization of the whole theory of Fourier series to the particular class of series in (26) if not indeed to the more general class involved in Theorem 20.

Thus we have an indication of an important and extended investigation where the results already known, while interesting and important, do little more than set the general problem awaiting solution.

11. Generalizations of the Preceding Section. Suggestions of various generalizations of the theory sketched in the preceding section come readily to mind. That theory is intimately based upon the function $h(t)$ in (21) and on special cases of it. The function $h(t)$ is of exponential type. The question arises whether there are other functions of exponential type, each having an infinitude of zeros, which might replace $h(t)$ in the theory. That the answer is affirmative seems almost certain, though the relevant investigation has not been carried out. Different classes of functions of exponential type may well give rise to different expansion theories. The whole problem is deserving of a thorough investigation.

It should be possible to extend the Birkhoff expansion theory along lines parallel to the extension of Fourier series indicated in the preceding section and in such a way that the natural regions of convergence of the resulting series shall be twodimensional rather than one-dimensional. Nothing has been done in the direction of this extension.

Any expansion theory of functions of a single variable, developed by such means as are indicated in the preceding sec- 
tion, is readily extensible to the case of functions of any finite number of variables. While no striking novelties are to be expected in this theory it is nevertheless worth while to have the theory developed.

12. Two Ways of Scientific Advance. In the preceding sections we have ample indications of the fact that the theory of functions of exponential type has numerous connections with problems of importance and that many questions concerning them are still awaiting investigation. In conclusion we shall indicate briefly two ways of scientific advance which are suggested by the unanswered questions to which we have referred.

In one of the ways of investigation we treat problems of just as general character as possible. Here the goal is a comprehensive theory. The purpose is to open up broad territories of investigation and to show the interconnections of related theories which have grown up separately. The problems are so general that they seldom lead to specific novelties of method. The solutions are likely to arise from extensions of known methods to wider problems.

The other method of investigation relies on the intense study of restricted problems. One lives with the ideas and questions involved until they become almost incorporated into his personality. Here lies the greatest hope of genuine novelty of idea and method, of discoveries which will prompt wide-reaching investigations. Intensive study of functions of exponential type would be a case in point. Another illustration would be afforded by the work of Stieltjes in developing his integral.

We need to cultivate both methods of investigation. We must have comprehensive theories. We should also live intimately with restricted problems. The suggestions which we have made concerning functions of exponential type are commended to the consideration of those who are interested in this second way of scientific advance.

The University of Illinois 